\title{
LINEAR DIFFERENCE AND DIFFERENTIAL EQUATIONS SATISFYING CONDITIONS AT MORE THAN ONE POINT ${ }^{1}$
}

TOMLINSON FORT

1. Introduction. The problem of differential equations satisfying conditions at more than one point is not new. W. M. Whyburn ${ }^{2}$ has given an extensive bibliography to which the reader is referred. In the present paper sufficient conditions on the coefficients are given that a solution of a system of linear equations exist consisting of a set of functions some of which take on prescribed values at one point and others at other points. The reader is referred to Theorems II and I for precise statements. The approach to differential equations is through difference equations. It is realized that most mathematicians will probably regard the differential equations as the more interesting. However, the author regards difference equations as of interest in themselves and Theorem I as of equal interest with Theorem II. In a way it is more fundamental since, as in many other places, the facts for the differential equations are inferred from those for difference equations.

2. The difference equation. Consider the system of difference equations

$$
y_{\nu}(i+1)=\sum_{\mu=1}^{n} a_{\nu \mu}(i) y_{\mu}(i), \quad \nu=1, \cdots, n .
$$

Here $i$ is limited to integral values and the coefficients $a_{\nu \mu}(i)$ are defined when

$$
0 \leqq i<b .
$$

There is no gain in generality if $(2)$ is replaced by

$$
a \leqq i<b \text {. }
$$

By a solution of (1) we shall mean a set of functions $y_{\nu}(i)$ which satisfy (1) at all points of (2). It is well known and immediately proved that there exists one and only one solution of (1) having arbitrary prescribed values at 0 .

Presented to the Society August 27, 1957; received by the editors June 30, 1957 and, in revised form, September 3, 1957.

1 This research was supported by the United States Air Force through the Air Force Office of Scientific Research of the Air Research and Development Command under contract No. AF 18(603-23).

${ }^{2}$ Proceedings of the conference on differential equations, edited by L. B. Diaz and L. B. Payne, University of Maryland Bookstore, 1956, p. 1. 
Suppose we are given an integer $a$, where $0 \leqq a<b$. Begin with $y \nu(a)$ and calculate successively $y \nu(a+1), y \nu(a+2), \cdots, y \nu(k)$ by means of (1), $\nu=1,2, \cdots, n$. Then let

$$
\begin{array}{rlrl}
y_{\nu}(k) & =\sum_{\mu=1}^{n} A_{\nu \mu}(a, k) y_{\mu}(a), & & \\
D_{\nu}(k, \alpha, \beta) & =\operatorname{det} . a_{\alpha_{p} \beta_{q}}(k), & & p, q=1, \cdots, \nu, \\
H_{\nu}(a, k, \alpha, \beta) & =\operatorname{det} . A_{\alpha_{p} \beta_{q}}(a, k), & p, q=1, \cdots, \nu, \\
\left(\alpha_{\zeta}, \delta_{\theta}, \beta, \gamma, m\right) & =\sum_{\mu=1}^{m} a_{\alpha_{\zeta} \beta_{\mu}}(k) A_{\gamma \mu \delta_{\theta}}(a, k), & \\
\left(\alpha_{\zeta}, \delta_{\theta}, m\right) & =\sum_{\mu=1}^{m} a_{\alpha_{\zeta} \mu}(k) A_{\mu \delta_{\theta}}(a, k), & \\
\Delta_{\nu}(a, k, \alpha, \beta, \gamma, \delta) & =\operatorname{det} .\left(\alpha_{p}, \delta_{q}, \beta, \gamma, \nu\right), & & \quad p, q=1, \cdots, \nu \\
& =D_{\nu}(k, \alpha, \beta) H_{\nu}(a, k, \gamma, \delta) . &
\end{array}
$$

Lemma. Hypothesis. $D_{\nu}(k, \alpha, \beta)>0$ whenever $a \leqq k<c$ and $\alpha_{1}<\alpha_{2}<\cdots<\alpha_{\nu}, \beta_{1}<\beta_{2}<\cdots<\beta_{\nu}$.

Conclusion:

(8) $H_{\nu}(a, c, \gamma, \delta)>0$ whenever $\gamma_{1}<\gamma_{2}<\cdots<\gamma_{\nu}, \delta_{1}<\delta_{2}<\cdots<\delta_{\nu}$.

Proof is by means of mathematical induction.

To begin with

$$
H_{\nu}(a, a+1, \alpha, \beta)=D_{\nu}(a, \alpha, \beta)>0 .
$$

This is the first step in the induction.

We shall next prove that

$$
H_{\nu}(a, k+1, \alpha, \beta)=\sum_{\eta_{1}, \cdots, \eta_{\nu}} D_{\nu}(k, \alpha, \eta) H_{\nu}(a, k, \eta, \beta)
$$

where the summation is taken for all possible combinations of $\eta_{1}, \eta_{2}, \cdots, \eta_{\nu}$ subject to the restrictions $\eta_{1}<\eta_{2}<\cdots<\eta_{\nu}$.

Proof is by straightforward verification.

From (3) we have

$$
y_{\nu}(k+1)=\sum_{\mu=1}^{n} A_{\nu \mu}(a, k+1) y_{\mu}(a) .
$$

Moreover 


$$
\begin{aligned}
y_{\nu}(k+1) & =\sum_{\mu=1}^{\nu} a_{\nu \mu}(k) y_{\mu}(k) \\
& =\sum_{\mu=1}^{n} a_{\nu \mu}(k) \sum_{\rho=1}^{n} A_{\mu \rho}(a, k) y_{\rho}(a) \\
& =\sum_{\mu=1}^{n} \sum_{\rho=1}^{n} a_{\nu \mu}(k) A_{\mu \rho}(a, k) y_{\rho}(a) \\
& =\sum_{\rho=1}^{n}\left[\sum_{\mu=1}^{n} a_{\nu \mu}(k) A_{\mu \rho}(a, k)\right] y_{\rho}(a) .
\end{aligned}
$$

Equating coefficients we have

$$
A_{\nu \rho}(a, k+1)=\sum_{\mu=1}^{n} a_{\nu \mu}(k) A_{\mu \rho}(a, k)=(\nu, \rho, n) .
$$

As a consequence of this and (5)

$$
H_{\nu}(a, k+1, \alpha, \beta)=\operatorname{det} .\left(\alpha_{p}, \beta_{q}, n\right), \quad p, q=1, \cdots, n .
$$

Note that although the determinant is of order $\nu$ each element is the sum of $n$ terms. We expand this determinant into $n^{\nu}$ determinants by columns thus

$$
\begin{aligned}
& H_{\nu}(a, k+1, \alpha, \beta)=\sum_{\eta_{1}, \cdots, \eta_{\nu}} \operatorname{det.~} a_{\alpha_{p} \eta_{q}}(k) A_{\eta_{q} \beta}(a, k), \\
& p, q=1, \cdots, \nu .
\end{aligned}
$$

Here the summation is extended to all possible combinations of $\eta_{1}, \eta_{2}, \cdots, \eta_{\nu}$, subject only to the restrictions $0<\eta_{j} \leqq n, j=1, \cdots, \nu$. We denote the determinants in (12) by

$$
D\left(\eta_{1}, \cdots, \eta_{v}\right) \text {. }
$$

We note that if any two $\eta$ 's are the same $D=0$. This is true since $D$ is then a determinant with two columns proportional. There remain $n(n-1) \cdots(n-\nu+1)=P$. determinants. We denote these by $D_{1}, \cdots, D_{P}$. In general $D_{i} \neq D_{j}$ if $i \neq j$. We note from (7) that

$$
\sum_{\eta_{1}, \cdots, \eta_{\nu}} D_{\nu}(k, \alpha, \beta) H_{\nu}(a, k, \eta, \beta)=\sum_{\eta_{1}, \cdots, \eta_{\nu}} \Delta_{\nu}(a, k, \alpha, \eta, \eta, \beta) .
$$

We now expand all determinants in the right hand member of (14) also by columns. Each of the determinants $D_{1}, \cdots, D_{P}$ occurs once and only once. Other determinants which occur in the expansion are zero having two columns proportional as explained above. We thus establish formula (9). Induction is now immediate and the lemma is proved. 
Theorem I. Let $0=k_{0}<k_{1}<\cdots<k_{q-1} \leqq b$ and $0<p_{1}<\cdots<p_{q}$ $=n$ be integers. Let $G_{1}, \cdots, G_{n}$ be arbitrary numbers. Suppose $D_{\nu}(k, \alpha, \beta)>0$ whenever $\alpha_{1}<\alpha_{2}<\cdots<\alpha_{\nu}, \quad \beta_{1}<\beta_{2}<\cdots<\beta_{\nu}$, $k_{j-1} \leqq k<k_{j}$ and $\nu=n-p_{j}, j=1, \cdots, q-1$. Then there exists one and only one solution of (1) such that

$$
\begin{gathered}
y_{p_{j+i}}\left(k_{j}\right)=G_{p_{j+i}}, \\
i=1, \cdots, p_{j+1}-p_{j}, j=0, \cdots, q-1, p_{0}=0, k_{0}=0 .
\end{gathered}
$$

To prove this we first fix $y_{1}(0)=G_{1}, \cdots, y_{n}\left(k_{q}\right)=G_{n}$. With this done we shall show that under the hypotheses of the theorem it is possible to determine $y_{1}(0), y_{2}(0), \cdots, y_{n}(0)$ in one and only one way, hence to determine one and only one solution with the prescribed values.

We write down (3) for $\nu=p_{1}+1, \cdots, n$ and $k=k_{1}$. We know by the lemma that $H_{n-p_{1}}\left(0, k_{1}, \alpha, \beta\right)>0$. Hence we can solve for $y_{p_{1}+1},(0), \cdots, y_{n}(0)$ in terms of $y_{p_{1}+1}\left(k_{1}\right), \cdots, y_{n}\left(k_{1}\right)$. We note that $y_{p_{1}+1}\left(k_{1}\right), \cdots, y_{p_{2}}\left(k_{1}\right)$ have known values namely $G_{p_{1}+1}, \cdots, G_{p_{2}}$. We write

$$
y_{\nu}(0)=f_{\nu}^{(1)}\left[G_{1}, \cdots, G_{p_{2}}, y_{p_{2}+1}\left(k_{1}\right), \cdots, y_{n}\left(k_{1}\right)\right], \quad \nu=1, \cdots, n .
$$

We now write (3) for $k=k_{2}, a=k_{1}, \nu=p_{2}+1, \cdots, n$. We solve these equations for $y_{p_{2}+1}\left(k_{1}\right), \cdots, y_{n}\left(k_{1}\right)$ in terms of $y_{p_{2}+1}\left(k_{2}\right), \cdots, y_{n}\left(k_{2}\right)$. We can do this since $H_{n-p_{2}}\left(k_{1}, k_{2}, \alpha, \beta\right)>0$ by the lemma. We substitute these values in (15) noting that

We write

$$
y_{p_{2}+1}\left(k_{2}\right)=G_{p_{2}+1}, \cdots, y_{p_{3}}=G_{p_{3}} .
$$

$$
y_{\nu}(0)=f_{\nu}^{(2)}\left[G_{1}, \cdots, G_{p_{3}}, y_{p_{3}+1}\left(k_{2}\right), \cdots, y_{n}\left(k_{2}\right)\right], \quad \nu=1, \cdots, n .
$$

We solve for $y_{p_{3}+1}\left(k_{2}\right), \cdots, y_{n}\left(k_{2}\right)$ in terms of $y_{p_{2}+1}\left(k_{3}\right), \cdots, y_{n}\left(k_{3}\right)$ and substitute in (16) getting

$$
y_{\nu}(0)=f_{\nu}^{(3)}\left[G_{1}, \cdots, G_{p_{4}}, y_{p_{4}+1}\left(k_{3}\right), \cdots, y_{n}\left(k_{3}\right)\right], \quad \nu=1, \cdots, n .
$$

We continue this process until we finally have

$$
y_{\nu}(0)=f_{\nu}^{(q-1)}\left[G_{1}, \cdots, G_{n}\right]
$$

and the theorem is proved.

3. The differential equation. Consider the set of differential equations 


$$
\frac{d y_{\nu}}{d x}=\sum_{\mu=1}^{n} g_{\nu \mu}(x) y_{\nu}, \quad \quad \nu=1, \cdots, n
$$

where $g_{\nu \mu}(x)$ are continuous.

$$
0 \leqq x \leqq 1 .
$$

Assume $h>0$. Let $R_{\nu \nu}(x)=1+h g_{\nu v}(x)$ and $R_{\nu \mu}(x)=h g_{\nu \mu}(x), \nu \neq \mu \mu$. Let

$$
D_{\nu}(x ; \alpha, \beta)=\operatorname{det} . R_{\alpha_{r} \beta s}, \quad r, s=1, \cdots, \nu
$$

and assume $\alpha_{1}<\alpha_{2}<\cdots<\alpha_{\nu} ; \beta_{1}<\beta_{2}<\cdots<\beta_{\nu}$.

Theorem II. Let $0=k_{0}<k_{1}<\cdots<k_{q} \leqq 1$. Let $0<p_{1}<p_{2}<\cdots$ $<p_{q}=n$ be integers and let $G_{1}, G_{2}, \cdots, G_{n}$ be arbitrary numbers. Suppose for sufficiently small $h D_{n-p_{j}}(x, \alpha, \beta)>0$ when $k_{j-1} \leqq x<k_{j}$, $j=1, \cdots, n$. Then there exists one and only one solution of (17) such that

$$
\begin{array}{rr}
y_{p_{j}+i}=G_{p_{j}+i} ; & p_{0}=0, k_{0}=0, \\
i=1, \cdots, p_{j+1}-p_{j} ; j=0, \cdots, q-1 .
\end{array}
$$

Proof will be made to depend upon the corresponding theorem for difference equations.

Assume $x_{i}$ so chosen that $x_{i} / h=i$ a positive integer or zero. Let

$$
b_{\nu \mu}(i)=g_{\nu \mu}(h i)=g_{\nu \mu}\left(x_{i}\right) .
$$

Consider the difference equations

$$
\frac{1}{h} \Delta \bar{y}_{\nu}(i)=\sum_{\mu=1}^{n} b_{\nu \mu}(i) y_{\mu}(i), \quad v=1, \cdots, n .
$$

We write these equations in the form

$$
\bar{y}_{\nu}(i+1)=\sum_{\mu=1}^{n} a_{\nu \mu}(i) \bar{y}_{\mu}(i), \quad v=1, \cdots, n
$$

where $a_{\nu \mu}(i)=R_{\nu \mu}\left(x_{i}\right)$ that is $a_{\nu \mu}(i)=h b_{\nu \mu}(i), \mu \neq \nu$ and

$$
a_{\nu \nu}(i)=1+h b_{\nu \nu}(i) .
$$

We expect to consider (18) when $h$ approaches 0 . In place of $h i$ we have written $x_{i}$. We also write $\bar{y}_{v}(i)$ or $\bar{y}_{v}\left(x_{i}\right)$ indiscriminately meaning exactly the same thing in each instance. Let $h$ be small. Mark the points $\left(x_{i}, \bar{y}_{\nu}\left(x_{i}\right)\right)$ in the Cartesian plane and connect them by straight line segments. We then write $\bar{y}_{\nu}(x)$ for the function defined by this graph. It is well known ${ }^{3}$ that if a solution of (17) satisfying conditions

${ }^{3}$ See, for example, Fort, Finite differences and difference equations in the real domain, Clarendon Press, Oxford, 1948, p. 164. 
$y_{1}(0)=c_{1}, y_{2}(0)=c_{2}, \cdots, y_{n}(0)=c_{n}$ is given and if a solution of (18) is determined satisfying $\bar{y}_{1}(0)=c_{1}(h), \cdots, \bar{y}_{n}(0)=c_{n}(h)$ and if $\lim _{h \rightarrow 0} c_{\nu}(h)=c_{\nu}, \nu=1, \cdots, n$ then $\lim _{h \rightarrow 0} \bar{y}_{\nu}(x)=y_{\nu}(x)$ uniformly in $x, 0 \leqq x \leqq 1$.

We now consider (18). Suppose that $k_{1}, \cdots, k_{q-1}$ are points $x_{i}$ respectively as near as possible to $k_{1}, \cdots, k_{q-1}$. Then we determine $\bar{y}\left(x_{i}\right)$ so that at $k_{1}^{\prime}, \cdots, k_{q-1}^{\prime}$ it satisfies the conditions imposed by the theorem upon $y(x)$ at $k_{1}, \cdots, k_{q-1}$. This entails the determination of $\bar{y}_{1}(0), \cdots, \bar{y}_{n}(0)$ as explained in the proof of the Theorem I. The basic operation in each instance was Cramer's Rule for solving linear algebraic equations. In each case both numerator and denominator determinants approach limits and the limit of the denominator is positive. These facts follow from the following considerations. Formula (10) shows that $A_{\nu \rho}\left(k_{j-1}, k\right)$ as functions of $k$ with fixed $\rho=n-p_{j}$ and $k_{j-1}$ satisfy (1) where $\nu=1, \cdots, n$. The initial conditions are $A_{\nu \nu}\left(k_{j-1}, k_{j-1}\right)=1$ and $A_{\nu \rho}\left(k_{j-1}, k_{j-1}\right)=0$ when $\rho \neq \nu$. Consequently when $h$ approaches $0, A_{\nu \rho}\left(k_{j-1}, x_{i}\right)$ approaches a limit. Denominator determinants namely $H_{\nu}\left(k_{j-1}, k_{j}, \alpha, \beta\right)$ have each element an $A$ which approaches a limit. Consequently $H_{\nu}\left(k_{j-1}, k_{j}, \alpha, \beta\right)$ itself approaches a limit. As a matter of fact $H_{\nu}\left(k_{j-1}, k, \alpha, \beta\right)$ as functions of $k$ satisfy a set of equations (9) which are a precise analogue of (1). Each of the $H^{\prime}$ 's then has a limit which is a member of a solution of a set of linear differential equations with positive coefficients and positive initial conditions. It is then positive as remarked. All other operations used in obtaining $\bar{y}_{1}(0), \cdots, \bar{y}_{n}(0)$ are a finite number of additions and multiplications of functions which approach limits. Hence $\bar{y}_{1}(0), \cdots, \bar{y}_{n}(0)$ approach $y_{1}(0), \cdots, y_{n}(0)$ and the corresponding solution satisfies the conditions of the theorem, since as we have remarked $k_{j}^{\prime} \rightarrow k_{j}, j=1, \cdots, q-1$.

University of South Carolina 